\title{
Health Issues for Psychosocial Counselling in Children Hector CHIBOOLA*
}

\author{
Faculty of Counselling Studies, Kabwe University, Zambia \\ *Corresponding author: hectorchiboola@yahoo.com
}

\begin{abstract}
Keywords: Child Health, Psychosocial Counselling, Interventions, Health Psychology.
\end{abstract}

\begin{abstract}
This article was developed based on the literature review with the intention of isolating health issues which affect children aged between 0-5 years and how these issues can inform the psychosocial needs in child counselling. Counselling children is a growing area of interest in contemporary society, especially amongst members of the counselling profession. Developmental psychologists have studied children's growth and development focusing on the effect of childhood experiences in adulthood, whereas child psychiatrists have focused on mentally disturbed children. It is apparent that children with learning, social, or behavioural problems who are not classified as mentally disturbed have been largely overlooked. These represent the target clientele for psychosocial counselling. Child counselling can prevent some problem situations from becoming more serious through degeneration to delinquency, school failure, antisocial conduct, and emotional disturbance.
\end{abstract}

\section{Introduction}

This article discusses the concept of health issues in the context of child counselling. The focus is on considering some selected disease conditions that adversely affect children below the age of five years that would require formal psychosocial counselling with regard to aspects of prevention, information, education, adherence to treatment and supportive care. Since this article is not for training health workers, great emphasis is placed on highlighting some selected disease conditions in underfive children that have an interface with child counselling from a psychosocial perspective. In 1946 the World Health Organization (WHO) defined health as: 'the state of complete physical, social and spiritual wellbeing, not simply the absence of disease or infirmity'. While it is difficult to understand how a person can achieve 'complete physical, social and spiritual wellbeing', it can be easily postulated that health is synonymous to disease. The traditional perspective suggests that health is not the opposite of disease, but it is a correlate to sickness. The modern understanding of health is that it integrates more factors than those advanced by the WHO. Health is a state of wellbeing with physical, cultural, psychosocial, socioeconomic, educational and spiritual aspects, not merely the absence of illness [1]. This is a broader definition which suggests that a 'health status' is better measured on a continuum of wellness and illness. Viewed from a wellness-illness continuum, on one end is optimal wellness and on the other is death, with the middle point being neutral health status. All human beings live, and eventually die.

Optimal wellness is dependent upon very healthful signs and lifestyle, that is, optimal balance in physical, cultural, psychosocial, socioeconomic, educational and spiritual needs. Death is a terminal state which occurs after a chronic illness, sudden injury or accident. The mid-point neutral health status integrates both parameters of wellness and illness that are contextually manageable and tolerable by degree of 
association, either at individual, family or group level. In this context, a person is regarded as enjoying 'good-enough health' even if he experiences occasional stress such as a traffic jam or mild headache due to a family discord. Equally, a person is not regarded as being in 'good-enough health' if his economic or educational needs are not satisfactorily met for the daily living. Between the neutral health status and terminal illness, there are average signs and symptoms that can be managed to restore the status quo of neutral health status. This is the basic principle for treatment of illnesses, physical exercise and observance of nutritional intake, including health promotion and prevention of diseases. When the healing fails, then a person advances to major disability from illness and eventually dies. Sedentary life precipitates physiological malfunctioning that may lead to illness. Obese people are not fundamentally healthy because their status renders them vulnerable to lifestyle diseases and psychological distress that could be fatal $[2,3]$. Children between the ages of 0-5 years are very prone to illness because their immune systems are not yet fully mature and their nutritional needs can easily be compromised. Because they have a habit of exploring the world around them with their hands (as they craw) and mouths (eat anything), this means that they come into contact with harmful elements in the process that can cause illness. Depending on the nature of illness and its prognosis however, most older-children have an inclination and resilience to quick recovery than adults; and most of them die from accidental injury than chronic diseases characteristic in adults $[4,5]$.

A person's optimum state of health is equivalent to the state of the set of conditions which fulfill or enable the person to achieve his realistic chosen and biological potentials. Some of these conditions are of the highest importance to all people, while others are variable and dependent upon individual abilities and potential. In other words, in addition to universal conditions (for all people) there are also specific conditions for different individual situations and circumstances. For instance, a person with a mental illness, a person with a physical disability, a person with a terminal disease, or an expectant mother will each have specific requirements, priorities, and environments [6]. A healthy environment provides safety, opportunities for social integration, and the ability to predict and/or control aspects of that environment; whereas an unhealthy environment threatens safety, undermines the creation of social ties, and is perpetually abusive or violent [7]. It is apparent that support and care are critical factors when considering health issues in children, primarily because children are dependent upon their parents, guardians, older siblings or relatives for survival in their daily life as well as when they are ill. Physical health and illness are inextricably interwoven with the psychological and social environments [8]. From this viewpoint, health becomes something that a person achieves through adequate attention to biological, psychological, sociocultural and environmental needs rather than something that is taken for granted [9]. The equation of wellness-illness is partly dependent upon variable factors as well as the people's perceptions and reactions.

Human beings are complex systems and illness can be caused by a multitude of factors, not just a single factor such as a virus or bacterium. A multiple combination of factors involved in illness are biological (virus, bacterium, lesion), psychological (behaviours, beliefs, attitudes) and social (literacy, poverty, unemployment), hence the biopsychosocial model of defining wellness and illness [10]. This broader view allows analyses, diagnosis and treatments of illnesses and injuries to incorporate multiple factors that might influence patients and their recovery, including improving the healing process [11]. The biopsychosocial model is integrative in orientation and looks at treating the person as a whole, that is, biologically, psychologically and 
socioculturally within his environmental context. In addition to treatment of any presenting physical illness, other measures include behaviour change, changes in beliefs and coping strategies, nutrition care, and adherence counselling for medical recommendations. The model emphasizes proactive interventions directed towards disease prevention, promotion of healthy behaviours and beliefs, prevention of accidental injury, and environment improvements [5]; and improved social relationships and support from family members and friends through enhanced psychosocial counselling [12].

The other interventions include changing people's lifestyles to prevent the onset of illness, and rehabilitation facilities; social support of resources provided by other people or an exchange of resources intended to enhance the well-being of child clients [13]; and maintaining close personal relationships with others which acts as a social resource factor that protects against illness and premature death. The psychosocial aspect of this model describes the way in which human behaviour and experiencing help to mold wellness and illness [1]. The role of a counsellor is to address the behavioural and social factors that impair the child client's normal functioning in society through systematic preventive counselling and health promotion. In chronic disease and related adverse conditions, the counsellor ought to provide supportive psychosocial counselling to the affected children through their parents or guardians [14].

\section{Selected Disease Conditions}

In developing countries, every year several millions of children die before they reach their fifth birthday, many during the first year of life; and eight in ten of these deaths are due to neonatal conditions, pneumonia, measles, diarrhoea, malaria and severe malnutrition, or a combination of these diseases [15]. Projections based on the 1996 analysis the global burden of disease indicate that these disease conditions will continue to be major contributors to child deaths in the year 2020 and beyond unless significantly greater efforts are taken to prevent and control them. In Zambia for instance, thousands of parents take their children with potentially fatal illnesses to first-level health facilities such as clinics, health centres and outpatient departments of local hospitals every day. In some countries, three in four episodes of childhood illness are caused by one of the referenced diseases; and most sick children present with signs and symptoms related to more than one disease [16]. This overlap means that a single diagnosis may not be possible or appropriate, and that treatment may be complicated by the need to combine medications for several different diseases. Anecdotal inferences on the management of sick children at health facility level reveal that many cases are not properly examined and treated, and their parents are poorly advised and guided, especially in rural communities where staffing and capacity of trained health personnel poses a gigantic challenge. It is in the context of this understanding that the need for psychosocial counselling in health becomes desirable, hence the appropriateness of this article.

Effective case management is only possible in situations where parents access early medical intervention for their sick children and counsellors provide proactive preventive counselling, supportive counselling and adherence counselling to the parents, caregivers and communities as a comprehensive continuum of care. The role of psychosocial counselling in this context is to: advise and guide parents on the importance of seeking early medical intervention, teaching and informing parents on how to care for a child at home, guidance to parents on how to solve feeding problems, 
advising parents when to return to the heath facility, and informing parents and communities on the importance of prevention and health promotion as conduits to optimal wellness. The selected health issues are: neonatal conditions, immunizable childhood diseases, pneumonia, diarrhoea, malaria, paediatric retroviral disease and malnutrition. These are discussed hereunder.

\section{Neonatal Conditions}

Neonatal conditions refer to health problems that affect infant children during their first month of life after birth (0-28 days). These include premature birth, neonatal infections and birth asphyxia. The first month of the infant's life is very critical because it accounts for a remarkable number of all deaths in children below the age of five years. Premature birth is defined as all births before 37 weeks of gestation or fewer than 259 days since the first day of a woman's last menstrual period. Complications of premature birth are the single largest contributor to neonatal deaths; and premature babies lack the necessary physical development which often requires special care and support. Survivors of premature birth may face greater risks of noncommunicable disease, and they may suffer lifelong effects such as impaired neurodevelopmental functioning, physical impairments in visual, hearing, lung and cardiovascular function [17].

Neonatal infections include disease conditions such as neonatal tetanus, sepsis, pneumonia, diarrhoea, and meningitis. Neonatal sepsis and meningitis are caused largely by bacterial germs that are transmitted to the infant baby by the mother during delivery. Having a catheter in a blood vessel and/or staying in the hospital for an extended period increases an infant's risk of sepsis after delivery. The chances of survival are reduced for infants with a serious infection regardless of whether or not they are hospitalized; and the complications of neonatal sepsis and meningitis may lead to death or lifelong disability. Birth asphyxia is the failure to establish breathing or perfusion at birth, and it is very fatal. It accounts for many premature neonatal deaths and complications of low oxygen intake include damage to the brain tissues that can cause seizures and other neurological problems, lifelong disability or death [17].

The primary agenda for psychosocial counselling related to neonatal conditions should be guidance and advocacy, based on the following key messages:

$>$ Risk factors for premature birth include age, multiple pregnancy, infection, maternal illness, psychological health and preparedness, nutritional, lifestyle and genetics. Mothers and fathers must be advised to dissuade their teenage female children from early marriage and pregnancy.

$>$ Expectant mothers and fathers must be advised on the importance of maternal health and antenatal clinics, as well as the need to seek early medical intervention when delivery is due.

$>$ Mothers and fathers must be advised on the importance of seeking early medical intervention for treatment of maternal illness to avoid mother-to-child transmission during delivery.

$>$ Mothers and fathers must be advised on the importance of family planning as an integral component of wellness at family level.

\section{Immunizable Childhood Diseases}

The WHO guidelines for vaccinating children through the expanded programme on immunization have been largely successful in ensuring that all children gain access to this service countrywide [18]. The service is offered routinely at under-five clinics 
and regularly through outreach programmes; whereas infant babies start accessing the service soon after birth at maternity clinics with the initial tuberculosis and poliomyelitis vaccinations. Immunization is the process of giving preventive medicine or vaccines by injection or orally to children $0-5$ years of age. The vaccines make the body produce antibodies that protect children (and adults) from getting a particular disease. Table 1 shows the common immunizable childhood diseases: tuberculosis (BCG), diphtheria, pertussis and tetanus (DPT), hepatitis B/haemophilus influenzae type b (HepB-Hib), poliomyelitis (polio), measles and rubella. Since the year 2006 in Zambia for instance, DPT is given as combination vaccine with HepB-Hib (DPTHepB-Hib); and in 2016 the Ministry of Health introduced a combination vaccine for measles and rubella. Neonatal tetanus is prevented by immunizing all expectant women with tetanus toxoid (TT). At least two dosages are recommended during the first pregnancy and three more each year or during each subsequent pregnancy. It might be of public health value to routinely vaccinate girl-children aged nine years and above with human papilloma virus (HPV) to prevent against cervical cancer in adulthood.

Immunizations should be started as soon as the child is born and be continued as scheduled until all the required dosages are given at the right times. Equally, repeat dosages must be given as indicated and, when desirable, children must be revaccinated without fail especially during disease outbreak. Immunizable childhood diseases are fatal: they cause suffering, disability and death if acquired and remain untreated. Since they are preventable, it is the responsibility of all childbearing mothers and fathers to ensure that their children get fully protected from immunizable childhood diseases. In Africa generally and Zambia in particular, many children suffer from preventable and immunizable childhood diseases and, out of these, some usually die. The livelihood of children is anchored upon their parents, guardians or older siblings depending on varying situations and circumstances.

Table 1. Immunization schedule

\begin{tabular}{|c|c|c|c|c|}
\hline $\begin{array}{l}\text { When } \\
\text { Started }\end{array}$ & Vaccine & Dosage & Disease & Remarks \\
\hline At birth & BCG+ & 1 & Tuberculosis & $\begin{array}{l}\text { Revaccination at } 5-7 \\
\text { years. }\end{array}$ \\
\hline $\begin{array}{cc}\text { At } & 6 \\
\text { weeks } & \end{array}$ & $\begin{array}{l}\text { DPT-HepB- } \\
\text { Hib++ }\end{array}$ & 4 & $\begin{array}{l}\text { DPT-HepB- } \\
\text { Hib }\end{array}$ & $\begin{array}{l}-2^{\text {nd }} \text { and } 3^{\text {rd }} \text { dosage } \\
\text { given at } 4-6 \text { week } \\
\text { intervals. } \\
\text {-Revaccination at } 18 \\
\text { months. }\end{array}$ \\
\hline At birth & Polio+++ & 4 & Poliomyelitis & $\begin{array}{l}-2^{\text {nd }} \text { and } 3^{\text {rd }} \text { dosage } \\
\text { given at } 4-8 \text { week } \\
\text { intervals. } \\
\text {-Revaccination at } 18 \\
\text { months. }\end{array}$ \\
\hline $\begin{array}{l}\text { At } 9 \\
\text { months }\end{array}$ & $\begin{array}{l}\text { Measles- } \\
\text { Rubella++++ }\end{array}$ & 1 & $\begin{array}{l}\text { Measles } \quad \& \\
\text { Rubella }\end{array}$ & $\begin{array}{l}\text {-Presumed life long } \\
\text {-Revaccination as } \\
\text { directed or during } \\
\text { epidemic }\end{array}$ \\
\hline
\end{tabular}

+ Revaccination advisable if there is no scar

++ Four vaccines combined in one. Revaccination advisable during disease outbreak 
outbreak

+++ Four dosages are sometimes recommended with first dosage at birth

++++ Two vaccines combined in one. Revaccination advisable during disease

The primary agenda for psychosocial counselling related to immunizable childhood diseases should be educational and informational, based on the following key messages:

$>$ Incomplete dosage of any vaccine does not guarantee full protection of the child. Mothers and fathers must be encouraged to complete the immunizations as scheduled.

$>$ Each vaccination only works for a particular disease as reflected in the immunization schedule. Mothers and fathers must be encouraged to check with their health worker and ensure that all vaccinations are given to their children on time.

$>$ Children should receive booster dosages for polio and DPT-HepB-Hib at eighteen months or whenever necessary as indicated by the relevant authorities such as during immunization week, disease outbreak, or school health day. Mothers and fathers must be advised on the need to abide by such directives in order to promote good health for their children.

$>$ Delayed and irregular vaccinations may adversely affect the uptake of vaccines according to approved timeframes. Mothers and fathers must be encouraged to cooperate with health workers and develop positive attitudes that foster promotion of a common good for the children's health.

$>$ Mothers and fathers must be informed and encouraged to be vaccinated tetanus toxoid to prevent neonatal tetanus during pregnancy.

$>$ Communities must be sensitized and provided information on the importance of prevention of immunizable childhood diseases. The child counsellor must work cooperatively and collaboratively together with individuals and families in the sensitization process through preventive counselling.

$>$ The expanded programme on immunization must be adequately supported and promoted as a viable intervention in child health.

\section{Pneumonia}

Pneumonia is a form of acute respiratory tract infection (ARTI) that affects the lungs and presents with severe chest pain, difficulty breathing, and dry cough, amongst many other symptoms. Pneumonia is the single leading cause of child deaths in every region of the world, with most deaths occurring in sub-Saharan Africa and South Asia [19]. Pneumonia is mainly caused by bacteria and viruses, and it is preventable through immunization with Haemophilus influenzae type $b$ vaccine [20]. Pneumonia can affect all age groups in a community, but children up to the age of five years and adults above 65 years are more susceptible to infection. Additionally, people suffering from chronic heart and lung disease, sickle cell anaemia, and retroviral disease are also at increased risk for pneumonia. Whereas healthy children have many natural defenses that protect them, undernourished children are at a higher risk of developing pneumonia.

The primary agenda for psychosocial counselling related to childhood pneumonia should be educational and informational, based on the following key messages:

$>$ Pneumonia is preventable. Mothers and fathers must be advised on the importance of immunization for pneumonia. 
Mothers and fathers must be advised on the need to avoid overcrowding children in sleeping rooms.

Mothers and fathers must be encouraged on the importance of seeking early medical treatment and adherence to the care and treatment plan for their sick children

\section{Diarrhoea}

Diarrhoea is defined as having loose or watery stool at least three times per day, or more frequently than normal for an individual. Although most episodes of childhood diarrhoea are mild, acute cases can lead to significant fluid loss and dehydration, which may result in death or other severe consequences if fluids are not replaced systematically. Diarrhoea is a common symptom of gastrointestinal infections caused by many germs such as bacteria, protozoa and viruses. Rotavirus is the leading cause of acute diarrhoea, and it is responsible for almost 40 percent of all hospital admissions to diarrhoea amongst children under-five years of age worldwide [21]. Most diarrhoeal germs are spread from the stool of one person to the mouth of another through contaminated water, food, or objects.

There are three main types of acute childhood diarrhoea, all of which are potentially life-threatening and require different treatment regimens: acute watery diarrhoea (including cholera) is associated with significant fluid loss and rapid dehydration in an infected individual; bloody diarrhoea (also referred to as dysentery) is marked by visible blood in the stool; and persistent diarrhoea that lasts at least 14 days. Undernourished children and those with paediatric retroviral disease are more likely to develop persistent diarrhoea, which worsens their already compromised condition. Diarrhoea is a common manifestation of human immunodeficiency virus (HIV) infection in both children and adults due to deteriorating immune system and frequent attacks by opportunistic infections. Children with poor nutritional and health status as well as those living in poor environmental conditions are susceptible to severe diarrhoea and dehydration than healthy children. Diarrhoea can have a detrimental impact on childhood growth and cognitive development.

The primary agenda for psychosocial counselling related to childhood diarrhoea should be educational and informational, based on the following key messages:

$>$ Since exposure to diarrhoea-causing germs is frequently related to the use of contaminated water and to unhygienic practices in food preparation and disposal of excreta (faecal matter), mothers and fathers must be advised on the importance of eating properly prepared food, drinking safe water, and living in clean environments.

Mothers and fathers must promote healthy behaviours and practices such as washing hands before eating food and after toilet, avoiding raw foods during diarrhoeal outbreaks, and avoiding open defecation. Disposal of children's faeces is equally very important.

$>$ Diarrhoeal episodes are frequently accompanied by vomiting which may adversely affect the child's appetite for food. Mothers and fathers must be advised on feeding practices in childhood diarrhoea.

$>$ Mothers and fathers must be encouraged to seek early medical intervention every time their children develop diarrhoea.

$>$ Communities must be sensitized and motivated to utilize available child health and care services, including the promotion of personal hygiene and environmental sanitation. 


\section{Malaria}

Malaria is caused by a parasite transmitted by female anopheles mosquitoes, which bite usually between dusk (early evening) and dawn (just before day break). The malaria-causing mosquitoes become active and troublesome during the night. For instance, there are plenty of mosquitoes in most parts of Zambia. This means that malaria is an endemic disease in most villages and communities, and both children and adults suffer from malaria occasionally. The initial symptoms of malaria are nonspecific and may be related to other infections such as headache, fatigue, abdominal pain, muscle and joint aches. Progressively they comprise fever, chills, perspiration, vomiting and worsening malaise; and in young children, malaria may also present with poor feeding and cough.

Malaria is easily preventable and treatable. However, if ineffective or poor-quality medicines are given or treatment delayed, the parasite burden continues to increase and the patient may develop severe malaria. Young children, expectant women and people who are immunosuppressed are particularly at risk of severe malaria; and expectant women with severe malaria are at increased risk of maternal death, miscarriage, stillbirth and neonatal death. Severe malaria usually manifests with one or more of the following: cerebral malaria, severe anaemia, acute renal failure or acute pulmonary oedema. In Zambia for instance, malaria accounts for up to 40 percent of all infant mortality and 20 percent of all maternal mortality [22]; and it poses a severe social and economic burden on people living in endemic communities [18]. Since malaria is an important contributory cause of death in infancy and childhood in many developing countries where malaria is endemic, presumptive treatment of fever with antimalarial medication is advocated because it is cost-effective.

The primary agenda for psychosocial counselling related to childhood malaria should be educational and informational, based on the following key messages:

$>$ Encourage individuals and families to be proactive in undertaking preventive activities such as cutting tall grass and emptying water in unusable containers within the surroundings of their homes, particularly during the rainy season.

$>$ Encourage individuals, especially young children and expectant women, to acquire and regularly use insecticide-treated mosquito nets during bedtime every day.

$>$ Encourage mothers and fathers to seek early medical intervention every time they notice signs of sickness in their children.

$>$ Encourage and advise mothers on the importance of feeding options for sick children.

\section{Paediatric Retroviral Disease}

Paediatric retroviral disease (HIV disease in children) is more aggressive, with a significant proportion of children presenting with symptoms in early infancy, and a small proportion presenting after infancy. Children with retroviral disease usually present with recurrent clinical episodes of common conditions such as pneumonia, persistent diarrhoea, and failure to thrive. They die in early childhood, most of them undiagnosed, particularly in rural communities with health provision challenges. Almost 95 percent of HIV infections in children are acquired through mother-to-child transmission during pregnancy, delivery or breastfeeding; and approximately slightly over one third of HIV-infected children die by the age of one year, and over half die by the age of two years [23]. Estimations of child morbidity and mortality due to paediatric retroviral disease keep increasing annually, suggesting that this problem will continue to erode many of the hard-worn gains in lowering rates of childhood illness and death, posing a public health threat. 
HIV infection in children is preventable through the intervention of prevention of mother-to-child transmission (PMTCT). Efforts to expand care and treatment for children ought to go hand-in-hand with efforts to rapidly improve the uptake of PMTCT services. High rates of maternal HIV infection, high birth rates, lack of access to recommended best practices, and the widespread practice of prolonged breast-feeding translate into a high burden of paediatric retroviral disease not only in Zambia, but many other countries in Africa as well. The fast rate of HIV progression and the high morbidity and mortality in children below five years with perinatally acquired HIV infection means that identifying these children and enrolling them in care and treatment programmes should be considered an emergency and of extreme importance as a public health requirement [24].

The primary agenda for psychosocial counselling related to paediatric retroviral disease should be educational and informational, based on the following key messages:

$>$ Encourage mothers and fathers to proactively seek and fully utilize PMTCT services at the right time (when they decide to have a baby) and for the duration of pregnancy (without defaulting).

$>$ Mothers and fathers must be adequately informed on the significance of adherence to the care and treatment plan in the context of PMTCT. Nonadherence affects drug efficacy.

$>$ Prolonged breast feeding increases the risk of acquiring HIV infection. Mothers must be encouraged to exclusively breast feed their infants for short periods or provide alternative feeding options when financial resources are available.

$>$ Paediatric retroviral disease is manageable through treatment. Mothers and fathers must be encouraged to access antiretroviral treatment (ART) for their infected children and themselves, as well as adhere to the prescribed care and treatment plan as appropriate.

$>$ Communities must be sensitized on the importance of universal preventive measures for HIV infection and care services for children.

$>$ Communities must advocate for the protection and support of children with or affected by paediatric retroviral disease.

\section{Malnutrition}

The term malnutrition refers to a deficiency of nutrition, that is, both to under nutrition (inadequate nutrition) and over nutrition (excessive nutrition) that result into illness. Many factors can cause malnutrition, most of which relate to poor diet or severe and repeated infections, prolonged illness, and inadequate food particularly in under privileged population groups globally [25]. Inadequate food and endemic disease are closely associated and linked to the general standard of living, the environmental conditions, and the capacity of individuals, families, communities and nationalities to meet their basic needs for livelihood and survival. Although it is rarely the direct cause of death (except in extreme famine situations), child malnutrition is associated with increased prevalence of child mortality in most developing countries presently, which is further compounded by worsening poverty and food inadequacy at community and family levels. Malnutrition is both a health outcome and a risk factor for disease, especially in children under five years of age. Although malnutrition commonly affects all age groups in a community, infants and young children are the most vulnerable because of their high nutritional requirements for growth and development and dependence on their parents, guardians or older siblings for provision of the needed food to enhance survival and nurturance. 
The nutritional status of women and children is very important, because it is through women and their children that the adverse effects of malnutrition are propagated to future generations. For instance, a malnourished expectant mother is likely to give birth to a low-birth-weight baby that would be susceptible to growth failure, impaired mental development, disease and premature death [26]. This compounds the economic development of the family and society, and perpetuates the intergenerational circle of poverty and malnutrition [27]. Adequate nutrition is essential in early childhood to ensure healthy growth, proper organ formation and function, a strong immune system, and neurological and cognitive development [28]. Child malnutrition adversely impacts cognitive functioning, contributes to poverty through impending individual's inability to lead a productive life, and contributes to a remarkable number of under-five deaths attributable to under nutrition.

Growth monitoring measures the growth level of a child on a monthly basis from birth until five years of age. The purpose of growth monitoring is to check whether or not the child is growing normally and healthily as well as to detect stuntedness (growth retardation) and underweight (failure to thrive). Underweight signifies that the child is either malnourished or diseased. When the child is not growing well or is losing weight, there is urgent need to redress this development because it could lead to fatality. It is apparent that access to good nutrition is a major and cross-cutting determinant of health. Presently, malnutrition underlies more than half of all underfive deaths, stunting rate in under-five children is close to half of all reported cases, and micronutrient deficiency rate is equally high [18]. Micronutrient deficiency contributes to functional anaemia and iodine deficiency disorders.

The primary agenda for psychosocial counselling related to childhood malnutrition should be educational and informational, based on the following key messages:

$>$ Malnutrition is closely linked to and has an effect on many other childhood diseases. Mothers and fathers must be encouraged to learn better ways of improving the family diet generally and that of their children specifically. Sharing information and experiences is a useful avenue in this regard.

$>$ Malnutrition is preventable. Mothers and fathers must be advised to give balanced foods in enough quantities and at the right times to their children every day. It is essentially necessary to promote and sustain the utilization of locally available foods through supportive counselling.

$>$ Mothers and fathers must be advised on the importance of growth monitoring as a barometer for detection of underweight or stunted growth. These have a symbiotic link with malnutrition.

$>$ Improving the child's diet might lead to appreciable weight gain and improved wellness. Mothers and fathers must be encouraged to seek early medical intervention when their children are losing weight, stunting, or sick.

Mothers and fathers must be encouraged to attend health education and promotional talks, including cooking demonstration sessions at health units or facilities when advised to do so by health workers.

\section{Conclusion}

It is commonly acknowledged that illness impairs the normal functioning of the body, soul and mind. For most children below the age of five years, illness is an alien anathema. They don't know what is wrong, what is happening, why they cannot eat, why they cannot play, why they are taking medicines, and so on. Most children are psychologically distressed when they are sick and this heightens their anxiety to get 
better soon. The longer they remain ill, the greater they become psychologically distressed. In turn, this adversely affects the parents and caregivers. Childhood illness evokes many psychosocial issues in both the children and parents. It is in the context of this understanding that psychosocial counselling becomes desirable and relevant. Child counselling must integrate a consideration of health issues that affect children in a wider context. Providing quality care to children suffering from the selected disease conditions can be a serious challenge and quite overwhelming. In response to this challenge, and in appreciation of the valuable role of curative care, there is need to promote and strengthen the management of childhood illness with aspects of nutrition, immunization, environmental hygiene and sanitation, prevention, health education and promotion, supportive care, and psychosocial counselling. The continuum of management and care should integrate the various aspects to enhance the children's wellness and livelihood as a public health requirement. Child counselling can prevent some problem situations from becoming more serious through degeneration to delinquency, school failure, antisocial conduct, and emotional disturbance.

\section{References}

[1] Marks, D. F., et al., Health psychology: Theory, research and practice, $3^{\text {rd }}$ edition. London: Sage. (2010).

[2] Braisby, N., \& Gellatly, A., Cognitive psychology, $2^{\text {nd }}$ edition. New York: OUP. (2012).

[3] Schneiderman, N., Antoni, M. H., Saab, P. G., \& Ironson, G., Health psychology: Psychological and behavioural aspects of chronic disease management. In: Annual Reviews Psychology, 52, 555-580, (2001).

[4] Costello, E., Mustillo, S., Erkanli, A., Keeler, G., \& Angold, A., Prevalence and development of psychiatric disorders in childhood and adolescence. In: Arch Gen Psychiatry, Vol. 60, 837-844. (2003).

[5] Sarafino, E. P., \& Smith, T. W., Health psychology: Biopsychosocial interactions, $7^{\text {th }}$ edition. London: Wiley. (2011).

[6] Berns R. M., Child, family, school, community: Socialization and support, $8^{\text {th }}$ edition. Belmont, California: Wadsworth. (2010).

[7] Taylor, S. E., Repetti, R. L., \& Seeman, T., Health psychology: What is an unhealthy environment and how does it get under the skin? In: Annual Review Psychology, 48, 411-447. (1997).

[8] Walker, J., Control and the psychology of health: Theory, measurement and applications. Buckingham: OUP. (2002).

[9] Taylor, S. E., Health psychology, $7^{\text {th }}$ edition. New York: McGraw-Hill. (2008).

[10] Hewstone, M., Fincham, F. D., \& Foster, J., Psychology, $1^{\text {st }}$ edition. Oxford: Blackwell. (2005).

[11] Snooks, M., Health psychology: Biological, psychological and sociocultural perspectives. Jones \& Bartlett Publishers. (2009).

[12] Baron, R., et al., Social support and immune function among spouses of cancer patients. In: Journal of Personality and Social Psychology, 59, 344-352. (1990). 
[13] Pawlik, K., \& Rosenzweig, R., The international handbook of psychology. California: Sage. (2000). http:1/dx.doi.org/10.4135/9781848608399.n23.

[14] Jordans, M. J. D., Training handbook on psychosocial counselling for children in especially difficult circumstances: A trainer's guide, third edition. Kathmandu, Nepal: UNICEF. (2003).

[15] WHO, Recommendations for management of common childhood conditions: newborn conditions, dysentery, pneumonia, oxygen use and delivery, common causes of fever, severe acute malnutrition and supportive care. Evidence for Technical Update of Pocketbook Recommendations. Geneva. (2012).

[16] WHO, \& UNICEF. (2005). Handbook: IMCI Integrated management of childhood illness. Geneva, (2005).

[17] Setiawan, C., Priority medicines for Europe and the world: A public health approach to innovation. Background Paper BP 6.23: Neonatal conditions. (2013).

[18] CSO, et al., Zambia demographic and health survey 2007. Calverton, Maryland: CSO/MII. (2009).

[19] Tong, N., Priority medicines for Europe and the world: A public health approach to innovation. Background Paper BP 6.22: Pneumonia. (2013).

[20] UNICEF, Pneumonia and diarrhoea: Tackling the deadliest diseases for the world's poorest children. Geneva. (2012).

[21] UNICEF \& WHO, Diarrhoea: Why children are still dying and what can be done. Geneva. (2009).

[22] MOH, Malaria indicator survey 2008. Lusaka: Ministry of Health. (2008).

[23] USAID \& BASICS, Child health and paediatric HIV/AIDS: A brief overview and update. NY. (2007).

[24] Tindyebwa, D. et al., Handbook on paediatric AIDS in Africa, revised edition. ANECCA. (2006).

[25] Blossner, M., \& de Onis, M., Malnutrition: Quantifying the health impact at national and local levels. Environmental Burden of Disease Series, No. 12. (2005).

[26] Felitti, V. J., \& Anda, R., The relationship of adverse childhood experiences to adult medical disease, psychiatric disorders, and sexual behaviour: Implications for healthcare. In: R. Lanius, E. Vermetten, \& C. Pain (eds.), The Hidden Epidemic: The Impact of Early Life Trauma on Health and Disease (2009). http://www.acestudy.org/yahoo_site_admin/assets/docs/LaniusVernetten_FINAL_826-09.12892303.pdf.

[27] Hernandez, D., Changing family circumstances. In: R. P. Weissberg, et al., (eds.), Long-Term Trends in the Well-being of Children and Youth: Issues in Children's and Families' Lives, (pp.155-180). Washington, DC: Child Welfare League of America, (2003).

[28] UNICEF, WHO, \& WORLD BANK, Levels and trends in child malnutrition: Joint child malnutrition estimates. Geneva. (2012). 